\title{
Open orbits and the semiclassical dwell time
}

\author{
C. H. Lewenkopf $\dagger$ and R. O. Vallejos $\ddagger$ \\ $\dagger$ Instituto de Física, Universidade do Estado do Rio de Janeiro, \\ R. São Francisco Xavier 524, 20550-900 Rio de Janeiro, Brazil \\ $\ddagger$ Centro Brasileiro de Pesquisas Físicas, \\ R. Dr. Xavier Sigaud 150, 22290-180 Rio de Janeiro, Brazil
}

\begin{abstract}
The Wigner delay time is addressed semiclassically using the Miller's $S$ matrix expressed in terms of open orbits. This leads to a very appealing expression, in terms of classical paths, for the energy averaged Wigner time delay in chaotic scattering. The same approach also puts in evidence the semiclassical incapability to correctly assess the time delay higher moments. This limitation suggests that the use of the semiclassical approximation to quantify fluctuations in scattering phenomena, like in mesoscopic physics, has to be considered with great caution.
\end{abstract}


What is the time a quantum particle spends to traverse a scattering region? Reported superluminal wave propagation [1] and its relation to the fascinating and controversial problem of the "tunneling time" [2, 3, 4] brought renewed interest to the quantum (or wave) dwell time question in general. The status of this matter is very nicely discussed by a recent comprehensive and pedagogical review [5].

The present study is devoted to examine the delay time of a particle scattered by a chaotic potential [6, 7] in the absence of tunneling barriers. Here the semiclassical approximation is employed to take a fresh look over the Wigner time delay [8]. Starting directly from the Miller semiclassical $S$ matrix, a quite appealing expression for the average dwell time is obtained and some quite unexpected limitations to the semiclassical approximation are clearly revealed.

The Wigner-Smith time delay matrix [8, 9] is defined as

$$
Q_{a b}(E)=-i \hbar \sum_{c=1}^{N} S_{a c}^{*} \frac{\partial S_{c b}}{\partial E},
$$

where the scattering matrix $S$, that encodes all accessible information about the scattering process, is taken at the energy $E$. The sum in (11) runs over all $N$ open asymptotic scattering channels. The Wigner time delay $\tau_{W}(E)$ is then given as

$$
\tau_{W}(E)=\frac{1}{N} \operatorname{tr} Q(E) \text {. }
$$

In scattering processes large enhancements of $\tau_{W}(E)$, or long dwell times, correspond to narrow isolated resonances. In these situations, the Wigner time delay gives access to properties of individual quasi-bound states. In the opposite and also relevant case of overlapping resonances, information about individual states is lost. The fluctuations of $\tau_{W}(E)$ result from the coherent interference of many resonances. Here the scattering process is best characterized by suitable statistical measures of $\tau_{W}(E)$ averaged over energy windows containing many resonances. In chaotic systems $\tau_{W}$ displays universal fluctuations that can be described by the theory of random matrices. A very detailed discussion on various aspects on the statistical approach for the Wigner time delay can be found, for instance, in [10].

The semiclassical literature on the Wigner time delay is invariantly based on its relation to the level density [11, 12], as envisaged long time ago by Friedel [13, 14]. Hence, the resulting semiclassical $\tau_{W}$ depends solely on the properties of the periodic orbits trapped in the scattering region. The semiclassical calculated Wigner time fluctuations coincide with the random matrix results [15, 16] for $N \gg 1$, as they should.

It is also desirable to cast the semiclassical $\tau_{W}$ in terms of open classical orbits that spend a finite time in the scattering region, closer to the spirit of a scattering problem. This is the task pursued here. Equation (1) is directly evaluated using the Miller's semiclassical $S$-matrix [17], namely

$$
\widetilde{S}_{a b}(E)=\sum_{\mu(a \leftarrow b)} \sqrt{p_{\mu}(E)} e^{i \sigma_{\mu}(E) / \hbar},
$$

where the classical trajectories that start at channel $b$ and end at channel $a$ are labelled by $\mu(a \leftarrow b)$. Accordingly, $\sigma_{\mu}$ is the reduced action (with a Maslov phase included) 
and $p_{\mu}$ is the classical transition probability for going from $b$ to $a$ following the path $\mu$ 6]. Throughout this paper the wide tilde indicates quantities obtained by means of the semiclassical approximation. In the derivation of (3) the absence of tunneling barriers between the scattering and the asymptotic regions is implicit. Furthermore, the number of open channels $N$ must fulfill $N \gg 1$.

For the semiclassical Wigner time delay, energy variations in classical transition probabilities $p_{\mu}$ are negligible as compared to those in the actions $\sigma_{\mu}$, since the latter are measured in units of $\hbar$. Hence,

$$
\widetilde{\tau}_{W}=\frac{1}{N} \sum_{\mu, \nu} t_{\mu} \sqrt{p_{\mu} p_{\nu}} e^{i\left(\sigma_{\mu}-\sigma_{\nu}\right) / \hbar}
$$

where $t_{\mu}=\partial \sigma_{\mu} / \partial E$ is the classical time the particle spends to go from $b$ to $a$ through the path $\mu$. Here the sums are unrestricted and run over all classical trajectories that enter and leave the scattering region. It is worth stressing that, due to the semiclassical approximation, $\widetilde{\tau}_{W}$ is not manifestly real for any given value of energy $E$. In analogy to the unitarity deficit of the semiclassical $S$-matrix [18, it can be shown that the imaginary part of $\widetilde{\tau}_{W}$ is of subleading order in powers of $1 / N$. This spurious imaginary part can be easily eliminated by using, for instance,

$$
Q_{a b}^{\mathrm{sym}}(E)=-\left.i \hbar \sum_{c} \frac{d}{d \varepsilon}\left[S_{a c}\left(E+\frac{\varepsilon}{2}\right) S_{a c}^{*}\left(E-\frac{\varepsilon}{2}\right)\right]\right|_{\varepsilon=0}
$$

instead of $Q_{a b}$ defined by (11). Restricting the analysis to the leading order term in powers of $1 / N$, it is possible to insist with $Q_{a b}$. In addition to the simplicity, this strategy has the merit of exposing some of the semiclassical limitations.

The statistical analysis of $\widetilde{\tau}_{W}$ and its higher moments unveils system specific, as well as universal features [5]. Let us start discussing the energy averaged $\widetilde{\tau}_{W}$, namely

$$
\left\langle\widetilde{\tau}_{W}\right\rangle=\frac{1}{N} \sum_{\mu, \nu}\left\langle t_{\mu} \sqrt{p_{\mu} p_{\nu}} e^{i\left(\sigma_{\mu}-\sigma_{\nu}\right) / \hbar}\right\rangle .
$$

Here $\langle\cdots\rangle$ indicates an energy average taken over an energy window $\Delta E$ where the classical dynamics presents little changes, nonetheless comprising many resonances. To compute $\left\langle\widetilde{\tau}_{W}\right\rangle$ it is justified to neglect the energy dependence of the probabilities $p_{\mu}$ and use the diagonal approximation. The latter says that, in general, different orbits of a chaotic system are uncorrelated, and holds for trajectories with dwell times shorter than the Heisenberg time $\tau_{\mathrm{H}} \equiv h / \Delta$. Here $\Delta$ is the mean resonance spacing. Fortunately, without barriers, trajectories with $t$ exceeding $\tau_{H}$ are statistically negligible in the semiclassical regime of $N \gg 1$ [12. In the absence of system specific symmetries the diagonal approximation reads $\left\langle\exp \left[i\left(\sigma_{\mu}-\sigma_{\nu}\right) / \hbar\right]\right\rangle=\delta_{\mu \nu}$, yielding

$$
\left\langle\widetilde{\tau}_{W}\right\rangle=\frac{1}{N} \sum_{\mu, \nu} t_{\mu} \sqrt{p_{\mu} p_{\nu}} \delta_{\mu \nu}=\frac{1}{N} \sum_{\mu} p_{\mu} t_{\mu} \equiv \tau .
$$

This remarkable equation expresses the multichannel energy averaged Wigner time delay purely in terms of classical quantities: $\tau$ is the classical scattering region escape time obtained by averaging over all trajectories weighted by their transition probabilities. 
The energy average eliminates the quantum interference terms in the leading order. Equation (77) holds for perfect transmission, that is guaranteed in the semiclassical limit by the absence of tunneling barriers. The diagonal approximation is customarily justified only for chaotic systems. Hence, chaos is a key element to obtain (77). The validity of the diagonal approximation for other kinds of dynamics is unclear. Thus, despite of the simplicity and appeal of (17), a rigorous derivation of an expression of the same kind for integrable systems is still lacking.

This (semi)classical result raises an important question: Is $\left\langle\widetilde{\tau}_{W}\right\rangle=h /(N \Delta)$ consistent with the well-known exact identity $\left\langle\tau_{W}(E)\right\rangle=h /(N \Delta)$, that holds irrespective of the underlying classical dynamics [7]? In other words, is it possible to prove $\tau=h /(N \Delta)$ using just geometric arguments?

The equivalence between both relations can be shown for the special case of chaotic billiards connected to the scattering region by wave guides. The proof is straightforward. The average time between bounces $\tau_{\mathrm{b}}$ for an ergodic billiard is

$$
\tau_{\mathrm{b}}=\frac{\pi A}{P v} \quad \text { for } d=2 \quad \text { and } \quad \tau_{\mathrm{b}}=\frac{4 V}{A v} \quad \text { for } d=3
$$

where $v=\sqrt{2 E / m}$ is the particle velocity. In two dimensions $(d=2), A / P$ is the billiard surface to perimeter ratio. Likewise, $V / A$ is the volume to surface ratio for three-dimensional $(d=3)$ cavities. The above relations are exact. As discussed in [19], their rigorous derivation is long known by mathematicians. Simple physical heuristic arguments can also used to obtained (8), as shown in [20].

For ergodic billiards the relation between the scape time $\tau$ and the average bounce time $\tau_{\mathrm{b}}$ is

$$
\tau=\frac{P}{W} \tau_{\mathrm{b}} \quad \text { for } d=2 \quad \text { and } \quad \tau=\frac{A}{S} \tau_{\mathrm{b}} \quad \text { for } d=3
$$

where $W$ and $S$ are respectively the wave guide width in $d=2$ and its cross section area in $d=3$. The Weyl formula can be used twice to relate the geometry of the billiard to the mean resonance spacing $\Delta$ and the number of channels in the wave guides $N$, yielding

$$
\tau=\frac{1}{N} \frac{h}{\Delta}
$$

for both two and three dimensional systems, as previously announced.

Let us now use the "open orbits" semiclassical approximation to calculate the Wigner time delay autocorrelation function, namely

$$
C(\epsilon)=\left\langle\tau_{W}(E+\epsilon) \tau_{W}(E)\right\rangle_{E}-\left\langle\tau_{W}(E)\right\rangle^{2} .
$$

More explicitly, we compute

$$
\widetilde{C}(\epsilon)=\frac{1}{N^{2}} \sum_{\substack{a, b \\ c, d}} \sum_{\substack{\mu, \nu(a \leftarrow b) \\ \mu^{\prime}, \nu^{\prime}(c \leftarrow d)}} \sqrt{p_{\mu} p_{\nu} p_{\mu^{\prime}} p_{\nu^{\prime}}} t_{\mu} t_{\mu^{\prime}}\left\langle e^{\frac{i}{\hbar}\left(\sigma_{\mu}-\sigma_{\nu}+\sigma_{\mu^{\prime}}-\sigma_{\nu^{\prime}}\right)}\right\rangle e^{\frac{i}{\hbar}\left(t_{\mu}-t_{\nu}\right) \epsilon}-\left\langle\widetilde{\tau}_{W}(E)\right\rangle^{2},
$$

and compare it with the result obtained using either the stochastic approach [15, 16, or the "closed orbits" semiclassical theory [11. The analysis of the transition probabilities 
$p_{\mu}$ gives the answer almost immediately. For chaotic systems the transition probabilities follow the analogue of the Hannay-Ozorio de Almeida sum rule for open systems [21, 22]

$$
\sum_{t \leq t_{\mu} \leq t+\delta t} p_{\mu}=\frac{1}{N \tau} \mathrm{e}^{-t / \tau} \delta t
$$

where $\sum_{t \leq t_{\mu} \leq t+\delta t} p_{\mu}$ is the sum of all classical transition probabilities following the trajectories $\mu$ belonging to the a small time interval $[t, t+\delta t]$, where $\delta t$ is classically small. In (13) the sum has no restriction on channels. Hence, it is easy to verify that by replacing the sum by a time integral, the classical normalization (flux conservation) condition [6]

$$
\sum_{a=1}^{N} \sum_{\mu(a \leftarrow b)} p_{\mu}=1,
$$

is fulfilled. Note that (14) must hold irrespective of the system dynamics.

The semiclassical Wigner time delay autocorrelation function $\widetilde{C}(\epsilon)$ can be calculated using the diagonal approximation

$$
\left\langle e^{\frac{i}{\hbar}\left(\sigma_{\mu}-\sigma_{\nu}+\sigma_{\mu^{\prime}}-\sigma_{\nu^{\prime}}\right)}\right\rangle=\delta_{\mu \nu} \delta_{\mu^{\prime} \nu^{\prime}}+\delta_{\mu \nu^{\prime}} \delta_{\nu \mu^{\prime}}
$$

and the sum rule (13). First, the diagonal approximation contracts pairwise the summations over the orbit indices in (12). As a result $\left\langle\widetilde{\tau}_{W}(E)\right\rangle^{2}$ is cancelled and only a double sum over the paths remains to be evaluated. By grouping the paths with similar traversal times we then use the sum rule (13) to transform the summations over orbits into time integrals. The later immediately give

$$
\widetilde{C}(\epsilon)=\frac{\tau^{2}}{N^{2}} \frac{1}{\left[1+\left(\frac{\epsilon \tau}{\hbar}\right)^{2}\right]^{2}} .
$$

Since no special attention is payed to time-reversal symmetric paths, (16) represents the semiclassical correlation function for broken time-reversal symmetry. It is noteworthy that although $\widetilde{\tau}_{W}(E)$ is not purely real, the energy average eliminates the imaginary part of $\widetilde{C}(\epsilon)$. However, (16) is at odds with the random matrix result [15, 16, that reads

$$
C(\epsilon)=\frac{2 \tau^{2}}{N^{2}} \frac{1-\left(\frac{\epsilon \tau}{\hbar}\right)^{2}}{\left[1+\left(\frac{\epsilon \tau}{\hbar}\right)^{2}\right]^{2}},
$$

in the limit of $N \gg 1$ and perfect transmission. The agreement is not reestablished by just starting with a Wigner-Smith matrix $Q$ which in the semiclassical limit is manifestly Hermitian, as for instance $Q^{\mathrm{sym}}$ in (5). Neither the variance, nor the $\epsilon$ dependence of the Wigner time correlation function defined as $\widetilde{\tau}_{W}^{\text {sym }}=(1 / N) \operatorname{tr} Q^{\text {sym }}$ agrees with (17).

Related problems appear when the semiclassical approximation using open orbits is employed to obtain the average transmission through a cavity and its autocorrelation functions, quantities of central interest in mesoscopic quantum coherent electronic transport. In this case the standard semiclassical approach fails because the $S$ matrix is 
not unitary. As previously discussed [18] this problem is best characterized by noticing that

$$
\left\langle\left(\sum_{c=1}^{N}\left|\widetilde{S}_{a c}(E)\right|^{2}-1\right)\left(\sum_{d=1}^{N}\left|\widetilde{S}_{b d}(E)\right|^{2}-1\right)\right\rangle=\frac{\delta_{a b}}{N} \neq 0 .
$$

Although admittedly small, the lack of unitarity is of the same order as typical transmission correlation functions. These spurious "unitarity" fluctuations could perhaps be reduced by including higher order corrections in the individual semiclassical $S$ matrix elements. However, a correct description of the correlations among different matrix elements, neglected in the standard semiclassical approach, results to be a more practical way to proceed. In fact, for the specific case of transmission, the discrepancies between random matrix theory and the semiclassical approximation could be fixed by introducing proper semiclassical sum rules that impose unitarity [18].

The Wigner time delay problem does not seem to have such a simple solution. Here the shortcomings of the semiclassical approach are more severe. For instance, a direct inspection shows that both the variance and the functional dependence of $\left\langle\widetilde{\tau}_{W}(E+\epsilon) \widetilde{\tau}_{W}(E)\right\rangle$ and $\left\langle\widetilde{\tau}_{W}(E+\epsilon) \widetilde{\tau}_{W}^{*}(E)\right\rangle$ are very different. Unfortunately, even (5) which seemed very promising to eliminate the semiclassical spurious imaginary part feature is of little help: Although $\widetilde{\tau}_{W}^{\mathrm{sym}}(E)$ is manifestly real, $\widetilde{\tau}_{W}^{\mathrm{sym}}(E) \widetilde{\tau}_{W}^{\mathrm{sym}}(E+\epsilon)$ is not.

In distinction to the approach presented in this study, the "closed orbits" semiclassical approximation to the Wigner time delay is very successful to describe its fluctuations [11, 12. The reason is simple. The trace over $Q$ allows one to express the Wigner time delay as a density of states, see for instance [15]. This is a more convenient starting point for the semiclassical approximation: the density of states is manifestly real and since $Q$ was already traced the unitarity problems of $\widetilde{S}$ do not appear. As a result one keeps only the contributions of closed orbits trapped in the scattering region, loosing all information about the channels. The small price to pay is that the simple physical interpretation for the average $\widetilde{\tau}_{W}$ is missed. On the other hand, since numerous observables of interest in scattering problems do not involve traces over all channels, a more general solution is desirable.

It is important to mention that for some classes of cross-section correlations, the relation between random-matrix theory [23] and semiclassical results is in very close relation to the above discussion. As nicely addressed by Ref. 24], apparent discrepancies between the two approaches are successfully remedied by avoiding taking singular semiclassical limits. Unfortunately we do not see how to adapt such ideas to our problem: The scattering problem considered in [24] allows for the use of a "closed orbits"-like semiclassical approximation that is free of the unitarity problems discussed here.

In summary, our study shows that the semiclassical $S$ matrix leads to a very appealing expression, in terms of classical paths, for the energy averaged Wigner time delay in chaotic scattering. On the other hand, it also puts in clear evidence the semiclassical incapability to correctly assess the time delay higher moments. This limitation can be attributed to the spurious imaginary part of $\widetilde{\tau}_{W}$ and to the lack of 
unitarity of the $\widetilde{S}$ matrix. The unitarity problem is quite severe and not only specific to the object studied in this work. Hence, before this problem is circumvented, applications of the semiclassical approximation to quantify fluctuations in scattering phenomena, like in mesoscopic physics, have to be considered with great caution.

\section{Acknowledgments}

The authors thank CNPq and PRONEX (Brazil) for partial financial support.

\section{References}

[1] Wang L J, Kuzmich A, Dogaiu A 2000 Nature 406, 277

Steinberg A M Kwiat P G Chiao R Y 1993 Phys. Rev. Lett. 71708

[2] Büttiker M and Landauer R 1982 Phys. Rev. Lett. 491739

[3] Büttiker M 1983 Phys. Rev. B 26178

[4] Nussenzveig H M 2000 Phys. Rev. A 62042107

[5] de Carvalho C A A and Nussenzveig H M 2002 Phys. Rep. 36483

[6] Smilansky U 1991 Les Houches 1989 Session LII on Chaos and Quantum Physics ed M J Gianonni, A Voros and J Zinn-Justin (Amsterdam: North-Holland) pp 371-441

[7] Lewenkopf C H and Weidenmüller H A 1991 Ann. Phys. (N.Y.) 21253

[8] Wigner E P 1955 Phys. Rev. 98145

[9] Smith F T 1960 Phys. Rev. 118349

[10] Fyodorov Y V and Sommers H J 1997 J. Math. Phys. 381918

[11] Eckhardt B 1993 Chaos 3613

[12] Vallejos R O, Ozorio de Almeida A M and Lewenkopf C H 1998 J. Phys. A: Math. Gen. 314885

[13] Friedel J 1952 Philos. Mag. 43153

[14] Balian R and Bloch C 1974 Ann. Phys. (N.Y.) 85514

[15] Lehmann N, Savin D, Sokolov V and Sommers H-J 1995 Physica 86D 572

[16] Lehmann N, Savin D, Sokolov V and Sommers H-J 1995 Nucl. Phys. A582 223

[17] Miller W H 1975 Advances in Chemical Physics ed K. P. Lawley (New York: Wiley) Vol. 30 p 77

[18] Vallejos R O and Lewenkopf C H 2001 J. Phys. A: Math. Gen. 342713

[19] Chernov N 1997 J. Stat. Phys. 881

[20] Jarzynski C 1993 Phys. Rev. E 484340

[21] Kadanoff L P and Tang C 1984 Proc. Natl. Acad. Sci. USA 811276

[22] Hannay J H and Ozorio de Almeida A M 1984 J. Phys. A: Math. Gen. 173429

[23] Fyodorov Y V and Alhassid Y 1998 Phys. Rev. A 58 R3375 Alhassid Y and Fyodorov Y V 1998 J. Phys. Chem. 1029577

[24] Eckhardt B, Fishman S and Varga I 2000 Phys. Rev. E 627867

Eckhardt B, Varga I and Pollner P 2001 Physica E 9535 